University of Nebraska - Lincoln

DigitalCommons@University of Nebraska - Lincoln

4-1-1976

\title{
EVIDENCE FAVORING NONEVOLUTIONARY CEPHEID MASSES
}

Norman R. Simon

University of Nebraska - Lincoln, nsimon@unl.edu

Edward G. Schmidt

University of Nebraska-Lincoln, eschmidt1@unl.edu

Follow this and additional works at: https://digitalcommons.unl.edu/physicsschmidt

Part of the Physics Commons

Simon, Norman R. and Schmidt, Edward G., "EVIDENCE FAVORING NONEVOLUTIONARY CEPHEID MASSES" (1976). Edward Schmidt Publications. 9.

https://digitalcommons.unl.edu/physicsschmidt/9

This Article is brought to you for free and open access by the Research Papers in Physics and Astronomy at DigitalCommons@University of Nebraska - Lincoln. It has been accepted for inclusion in Edward Schmidt Publications by an authorized administrator of DigitalCommons@University of Nebraska - Lincoln. 
The Astrophysical Journal, 205:162-164, 1976 April 1

(C) 1976. The American Astronomical Society. All rights reserved. Printed in U.S.A.

\title{
EVIDENCE FAVORING NONEVOLUTIONARY CEPHEID MASSES
}

Norman R. Simon and Edward G. SchmidT

Behlen Observatory and Behlen Laboratory of Physics, University of Nebraska, Lincoln

Received 1975 June 16; revised 1975 September 11

\begin{abstract}
It is suggested that the bumps on the velocity curves of nonlinear Cepheid models may be understood as the consequence of a resonance between the fundamental and the second overtone modes of the pulsating star. Thus the presence of bumps can be inferred from linear calculations. The region of the observational instability strip containing bumps is compared with the location of the calculated resonances. As was the case for the nonlinear calculations, we find that masses considerably less than evolutionary values are required to bring agreement between the observations and the theory.
\end{abstract}

Subject headings: stars: Cepheids - stars: evolution - stars: pulsation

I. CEPHEID BUMPS AND THE RESONANCE

$$
P_{2} / P_{0}=0.5
$$

The purpose of this section will be to establish a strong qualitative relationship between the bumps reported by Stobie $(1969 a, b, c)$ on his nonlinear theoretical velocity curves, and a fundamental-second overtone resonance $\left(P_{2} / P_{0} \approx 0.5\right)$ occurring in the linear adiabatic periods for the same models.

In the present investigation, the thermodynamics employed is that described in the Appendix of Stobie (1969a), while the opacities used are those given by the analytic formula of Christy (1966). The mechani- cal boundary condition is taken from Baker and Kippenhahn (1965) and is applied at the photosphere. Convection is neglected. Linear adiabatic periods are determined as described by Baker and Kippenhahn (1965), with integrations extending down to about 2 million degrees. The second overtone period $P_{2}$ is generally good to less than $\frac{1}{2}$ percent in either direction; the fundamental periods are considerably more accurate.

Results are given in Table 1 which refer to models investigated by Stobie (1969b). The first column gives Stobie's model number, except for the last six entries, where no such number exists; for these cases, the

TABLE 1

Bumps on Theoretical Velocity Curves (Stobie $1969 b$ ) Versus Resonance in THE Linear Adiabatic Periods

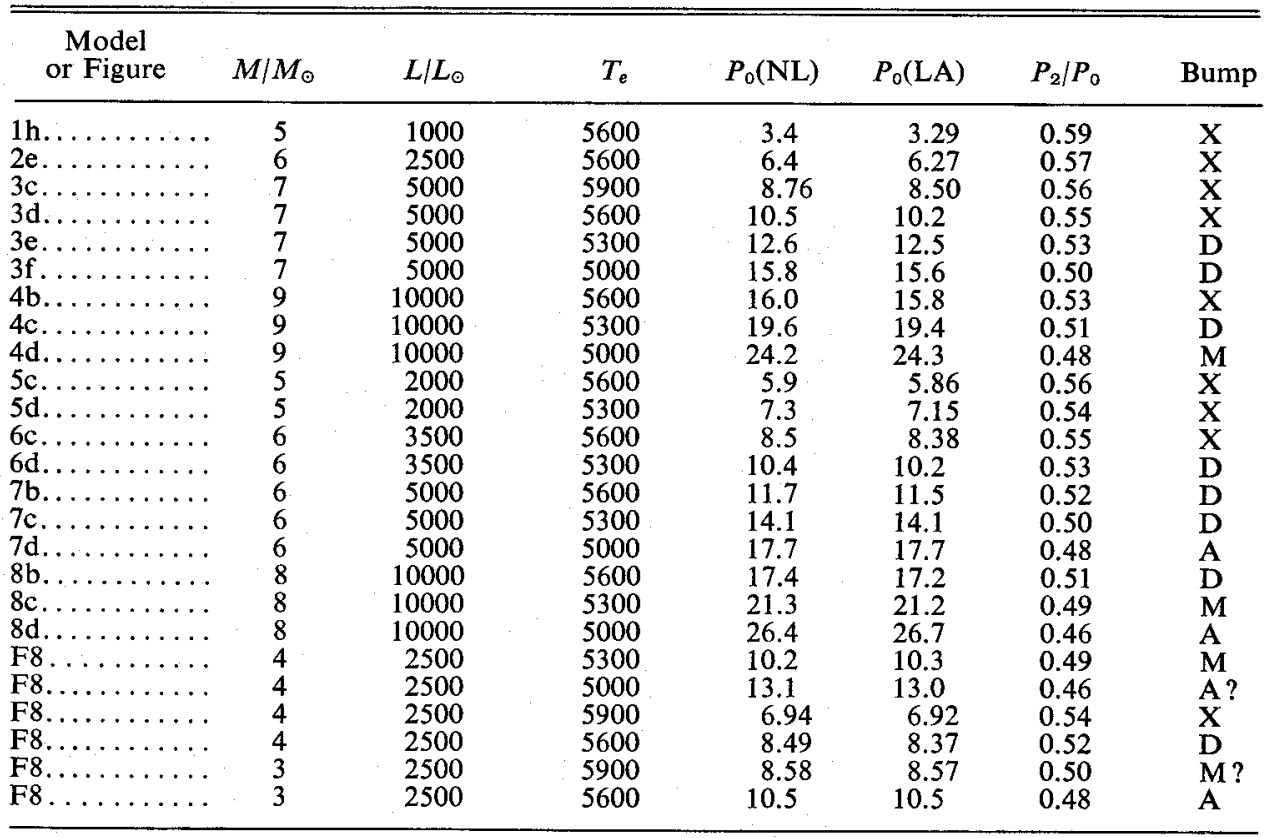


code "F8" refers to Figure 8 of Stobie $(1969 b)$, where data on the corresponding models are presented. Stobie's nonlinear fundamental periods are listed in the fifth column, and the corresponding linear adiabatic periods from the present investigation in the sixth column. The seventh column gives the ratio of the linear adiabatic periods, $P_{2} / P_{0}$. In the last column, the absence of a bump on Stobie's theoretical velocity curves is denoted by $\mathrm{X}$; if a bump appears, the designations $\mathrm{M}, \mathrm{D}$, and $\mathrm{A}$ indicate, respectively, its location at maximum, or on the descending, or ascending, branch of the theoretical curves. The bump designations for all but the last six entries are those of Stobie himself; for the cases marked F8, the designations are ours, based upon the plots appearing in Figure 8 of Stobie $(1969 b)$. The chemical composition is always $X=0.66, Y=0.30$, except for the F8 entries, for which the composition is given by Stobie in his Figure 8. It is important to note that Table 1 includes with a single exception every model in Stobie's (1969b) two surveys that had $X=0.66$, $Y=0.30$ and was unstable in the fundamental mode. The exception mentioned is model $4 \mathrm{~d}\left(T_{e}=4700\right)$, which, due to some bug in our computer program, could not be integrated.

A look at the last two columns of Table 1 suffices to show the striking relationship between the resonance $P_{2} / P_{0} \approx 0.5$ and the bumps on Stobie's theoretical velocity curves. In every case where a bump exists, $P_{2} / P_{0}$ is between 0.46 and 0.53 , whereas if $P_{2} / P_{0}$ falls on the high side of this range, there is no bump. Although the low end of the range is not well delineated by the data in Table 1, the weakness of the bump in the "F8" model $M / M_{\odot}=4, L / L_{\odot}=2500$, $T_{e}=5000$ (see Fig. 8 of Stobie 1969b) indicates that $P_{2} / P_{0} \approx 0.46$ constitutes the low boundary of the bump region. Furthermore, there is a definite connection between the location of the bump and the value of $P_{2} / P_{0}$. The $\mathrm{D}$ case always corresponds to $0.53 \geq P_{2} / P_{0} \geq 0.50$, while the A case corresponds to $0.48 \geq P_{2} / P_{0} \geq 0.46$.

The information in Table 1 suggests that the bumps in the velocity curves of nonlinear theoretical models are a consequence of the second overtone being driven by the fundamental in those cases where a nearresonance occurs. The driving of higher harmonics during pulsation has been studied by Simon (1972) and Simon and Sastri (1972), who have emphasized the pervasive influence of resonances on the harmonic structure of nonlinear oscillations. Simon and Sastri (1972) suggested that Cepheid pulsators might be classifiable according to the resonance characteristics of their linear normal modes.

On the other hand, Christy (1967) attributed the bumps in his nonlinear Cepheid velocity curves to the reflection of a pressure wave off the stellar core. This explanation was supported in a single case by Karp (1975), who nonetheless concluded that an alternate mechanism was required to reconcile certain of the observations. Fricke, Stobie, and Strittmatter (1972) pointed out that the reflected-wave theory does not explain why some stars exhibit strong bumps while others with large amplitudes do not. Furthermore, the high regularity observed among the bump Cepheids (see below) argues for a single underlying cause, such as might be provided by the resonance. The main shortcoming of the resonance hypothesis lies in the need to demonstrate that an unstable fundamental is able to drive and maintain pulsations at a period corresponding to that of the resonant overtone. This question can only be approached via further nonlinear calculations.

If it is indeed true that the resonance and the bump are causally related, then the bump structure of Stobie's velocity curves (and also those of Christy 1968) cannot be merely a numerical artifact attributable to coarse zoning (see, e.g., Keller and Mutschlecner 1971). On the contrary, this structure must be physically real. Thus the derivation of nonevolutionary Cepheid masses from the location of the bump should be essentially correct. This question is discussed further below.

\section{COMPARISON WITH OBSERVATIONAL DATA}

Employing a linear adiabatic code with the input physics described in $\S \mathrm{I}$, we have determined the location of resonance regions in the period-luminosity and temperature-luminosity diagrams. In accord with Table 1, the hot (short-period) boundary of the region is defined by models for which $P_{2} / P_{0}=0.53$. Since the cool (long-period) boundary is not as well determined by the data in Table 1 , we have taken it to be defined by $P_{2} / P_{0}=0.47$, so as to be symmetric about the resonance center. We have also determined the locus of models with $P_{2} / P_{0}=0.50$ in the two diagrams.

The calculations have been performed for evolutionary masses and for masses one-half the evolutionary values. The models are described in Table 2 , and the results plotted in Figure 1, where solid lines indicate the location of the resonance region for models of one-half evolutionary mass. The shortperiod boundary of the resonance region for models of evolutionary mass is given by the dashed lines. In the same diagram we have plotted data for the 41

TABLE 2

Models Defining Resonance Regions in FIGURE 1

\begin{tabular}{|c|c|c|c|c|}
\hline$M / M_{\odot}$ & $L / L_{\odot}$ & $T_{\text {eff }}$ & $P_{0}$ & $P_{2} / P_{0}$ \\
\hline \multicolumn{5}{|c|}{$M=M_{e v} / 2$} \\
\hline $\begin{array}{l}4 \ldots \ldots \ldots \\
4 \ldots \ldots \ldots \\
4 \ldots \ldots \ldots \\
3.5 \ldots \ldots \\
3.5 \ldots \ldots \\
3.5 \ldots \ldots \\
3.0 \ldots \ldots \\
3.0 \ldots \ldots \\
3.0 \ldots \ldots\end{array}$ & $\begin{array}{l}7500 \\
7500 \\
7500 \\
5000 \\
5000 \\
5000 \\
2500 \\
2500 \\
2500\end{array}$ & $\begin{array}{l}7200 \\
6600 \\
6200 \\
7000 \\
6400 \\
6000 \\
6400 \\
5900 \\
5500\end{array}$ & $\begin{array}{c}8.89 \\
12.2 \\
15.5 \\
7.56 \\
10.5 \\
13.4 \\
6.33 \\
8.55 \\
11.2\end{array}$ & $\begin{array}{l}0.53 \\
0.50 \\
0.47 \\
0.53 \\
0.50 \\
0.47 \\
0.53 \\
0.50 \\
0.47\end{array}$ \\
\hline \multicolumn{5}{|c|}{$M=M_{e v}$} \\
\hline $\begin{array}{l}9.0 \ldots \ldots \\
7.0 \ldots \ldots \\
6.0 \ldots \ldots\end{array}$ & $\begin{array}{r}10000 \\
5000 \\
2500\end{array}$ & $\begin{array}{l}5600 \\
5300 \\
5000\end{array}$ & $\begin{array}{c}15.8 \\
12.5 \\
9.46\end{array}$ & $\begin{array}{l}0.53 \\
0.53 \\
0.53\end{array}$ \\
\hline
\end{tabular}



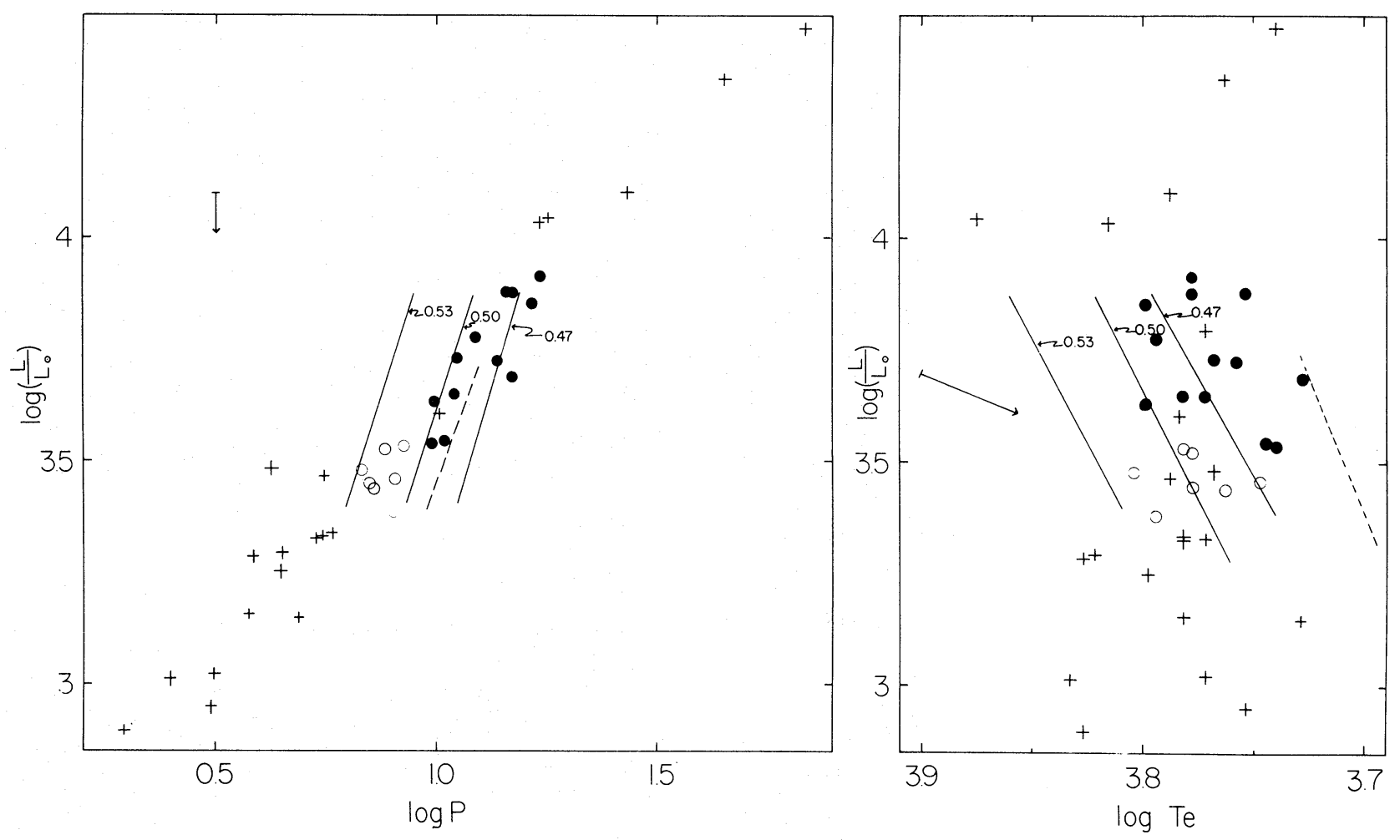

FIG. 1.-Period-luminosity and luminosity-temperature diagrams of the Cepheid region. Solid circles, Cepheids with the bump before light maximum (A case); open circles, those with a bump after maximum (D case); crosses, those without a bump. Solid lines, the location of the calculated resonance region for masses which are one-half of the evolutionary mass. They are labeled with $P_{2} / P_{0}$. Dashed line, the hot boundary $\left(P_{2} / P_{0}=0.53\right)$ of the resonance region for evolutionary mass. The arrows show the effect on the observed points of decreasing $E_{B-V}$ by $0.1 \mathrm{mag}$.

Cepheids for which $(R-I)$ photometry is available (Schmidt 1973, 1976). Crosses indicate stars for which there is no bump, while the circles indicate the presence of bumps-solid circles when the bump precedes light maximum (the A case), open circles when the bump follows light maximum (the $\mathrm{D}$ case). In the periodluminosity diagram, the data display great regularity. Not only are the A stars well separated from the D stars, but only a single cross appears among the circles. This star is $\zeta$ Gem.

It can be seen from Figure 1 that the resonance lines correspond well to the region of the instability strip containing the bump Cepheids if one-half evolutionary mass is assumed. This correspondence includes the approximate division of $\mathrm{D}$ from $\mathrm{A}$ Cepheids by the resonance line $P_{2} / P_{0}=0.50$. On the other hand, the evolutionary mass calculations fall far from the observed region. In this way we recover from the linear theory the conclusion of Christy (1968) and Stobie $(1969 b, c)$, that the location of bumps indicates low masses. If the suggested causal relationship between the resonance and the bumps can be confirmed by further investigation strong new evidence will thus have been provided supporting the contention that Cepheid masses are nonevolutionary.

One of us (N. R. S.) is happy to acknowledge informative interchanges with Richard Stothers, and an interesting conversation with Alan Karp which took place at the 146th Meeting of the American Astronomical Society.

\section{REFERENCES}

Baker, N., and Kippenhahn, R. 1965, Ap. J., 142, 868. Christy, R. F. 1966, Ap. J., 144, 108.

. 1967, in Aerodynamical Phenomena in Stellar Atmospheres, ed. R. N. Thomas (London: Academic Press), p. 105 .

P. 1968, Quart. J.R.A.S., 9, 13.

Fricke, K., Stobie, R. S., and Strittmatter, P. A. 1972, Ap.J., 171, 593.

Karp, A. H. 1975, Ap. J., 199, 448.
Keller, C. F., and Mutschlecner, J. P. 1971, Ap. J., 167, 127. Schmidt, E. G. 1973, M.N.R.A.S., 163, 67. . 1976, Ap. J., 203, 466.

Simon, N. R. 1972, Astr. and Ap., 21, 45.

Simon, N. R., and Sastri, V. K. 1972, Astr. and Ap., 21, 39.

Stobie, R. S. $1969 a$, M.N.R.A.S., 144, 461. $1969 b$, ibid., p. 485.
$.1969 c$, ibid., p. 511 .

Edward G. Schmidt and Norman R. Simon: Behlen Laboratory of Physics and Astronomy, University of Nebraska, Lincoln, NE 68508 